\title{
Pharyngeal Impairment During Swallowing
}

National Cancer Institute

\section{Source}

National Cancer Institute. Pharyngeal Impairment During Swallowing. NCI Thesaurus.

Code C127180.

An assessment of an individual's pharyngeal impairment during swallowing. 